\title{
Article \\ Biosynthesis and Heterologous Expression of Cacaoidin, the First Member of the Lanthidin Family of RiPPs
}

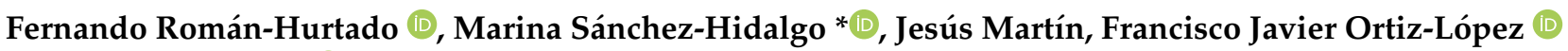 \\ and Olga Genilloud (1)
}

check for

updates

Citation: Román-Hurtado, F.; Sánchez-Hidalgo, M.; Martín, J.; Ortiz-López, F.J.; Genilloud, O. Biosynthesis and Heterologous Expression of Cacaoidin, the First Member of the Lanthidin Family of RiPPs. Antibiotics 2021, 10, 403. https://doi.org/10.3390/ antibiotics 10040403

Academic Editor: Jesus Simal-Gandara

Received: 22 March 2021

Accepted: 6 April 2021

Published: 8 April 2021

Publisher's Note: MDPI stays neutral with regard to jurisdictional claims in published maps and institutional affiliations.

Copyright: (c) 2021 by the authors. Licensee MDPI, Basel, Switzerland. This article is an open access article distributed under the terms and conditions of the Creative Commons Attribution (CC BY) license (https:/ / creativecommons.org/licenses/by/ $4.0 /)$.
Fundación MEDINA, Avenida del Conocimiento, 34, 18016 Granada, Spain; fernando.roman@medinaandalucia.es (F.R.-H.); jesus.martin@medinaandalucia.es (J.M.); javier.ortiz@medinaandalucia.es (F.J.O.-L.); olga.genilloud@medinaandalucia.es (O.G.)

* Correspondence: marina.sanchez@medinaandalucia.es

\begin{abstract}
Cacaoidin is produced by the strain Streptomyces cacaoi CA-170360 and represents the first member of the new lanthidin (class V lanthipeptides) RiPP family. In this work, we describe the complete identification, cloning and heterologous expression of the cacaoidin biosynthetic gene cluster, which shows unique RiPP genes whose functions were not predicted by any bioinformatic tool. We also describe that the cacaoidin pathway is restricted to strains of the subspecies Streptomyces cacaoi subsp. cacaoi found in public genome databases, where we have also identified the presence of other putative class $\mathrm{V}$ lanthipeptide pathways. This is the first report on the heterologous production of a class $\mathrm{V}$ lanthipeptide.
\end{abstract}

Keywords: cacaoidin; lanthidin; class V lanthipeptide; RiPP; Streptomyces cacaoi; heterologous expression

\section{Introduction}

Actinomycetes are an extremely diverse group of Gram-positive, filamentous bacteria with high GC content genomes [1] considered as one of the most prolific sources for the discovery of new natural products (NPs) [2,3]. Among all the actinomycetes, the genus Streptomyces produces over $70-80 \%$ of the secondary metabolites with described therapeutic properties [4].

The increasing number of sequenced genomes has revealed that actinomycetes carry the genetic potential to produce many more secondary metabolites than those detected under laboratory conditions [5]. The development of bioinformatic tools to identify the presence of new secondary metabolite Biosynthetic Gene Clusters (BGCs) from genome sequences, such as antiSMASH [6] or MIBiG [7], among others [8], has permitted the development of targeted genome mining strategies directed at variants of specific families of compounds [9]. Among them, Ribosomally synthesized and Post-translationally modified Peptides (RiPPs) show more structural diversity than initially predicted from genome sequences [10]. Most RiPPs are synthesized as a longer precursor peptide, containing a C-terminal core peptide that undergoes a broad diversity of Post-Translational Modifications (PTMs) directed by the N-terminal leader peptide [11].

We have recently described the discovery of cacaoidin, a novel glycosylated lantibiotic produced by the strain Streptomyces cacaoi CA-170360 [12]. Cacaoidin represents the first reported member of lanthidins, also known as the new class V lanthipeptides, exhibiting remarkable structural features, such as a lanthionine ring and N-terminal dimethylation, restricted thus far to lanthipeptide [13] and linaridin [14-16] RiPP families, respectively, in an unprecedented $N, N$-dimethyl lanthionine system $\left(\mathrm{NMe}_{2} \mathrm{Lan}\right)$ [12] (Figure 1). In addition, cacaoidin contains several PTMs, some of them shared with other lanthipeptides, such as a C-terminal S-[(Z)-2-aminovinyl-3-methyl]-D-cysteine (AviMeCys) amino acid. AviMeCys is formed in lanthipeptides through an oxidative decarboxylation of the C-terminal cysteine by LanD and the resulting reactive thio-enol intermediate cyclises with a Dhb residue, 
yielding AviMeCys [17]. This differs from the AviCys formation in linaridins [18], which occurs between two cysteine residues and is catalyzed by $\operatorname{LinD}$ [19]. The co-existing structural features restricted to lanthipeptides and linaridins have supported the proposal of cacaoidin as the first reported member of the lanthidins [12]. Cacaoidin also presents other unusual structural features, such as a high number of D-amino acids and an O-glycosylated tyrosine residue carrying a non-previously reported disaccharide formed by $\alpha$-L-rhamnose and $\beta$-L-6-deoxygulose [12].

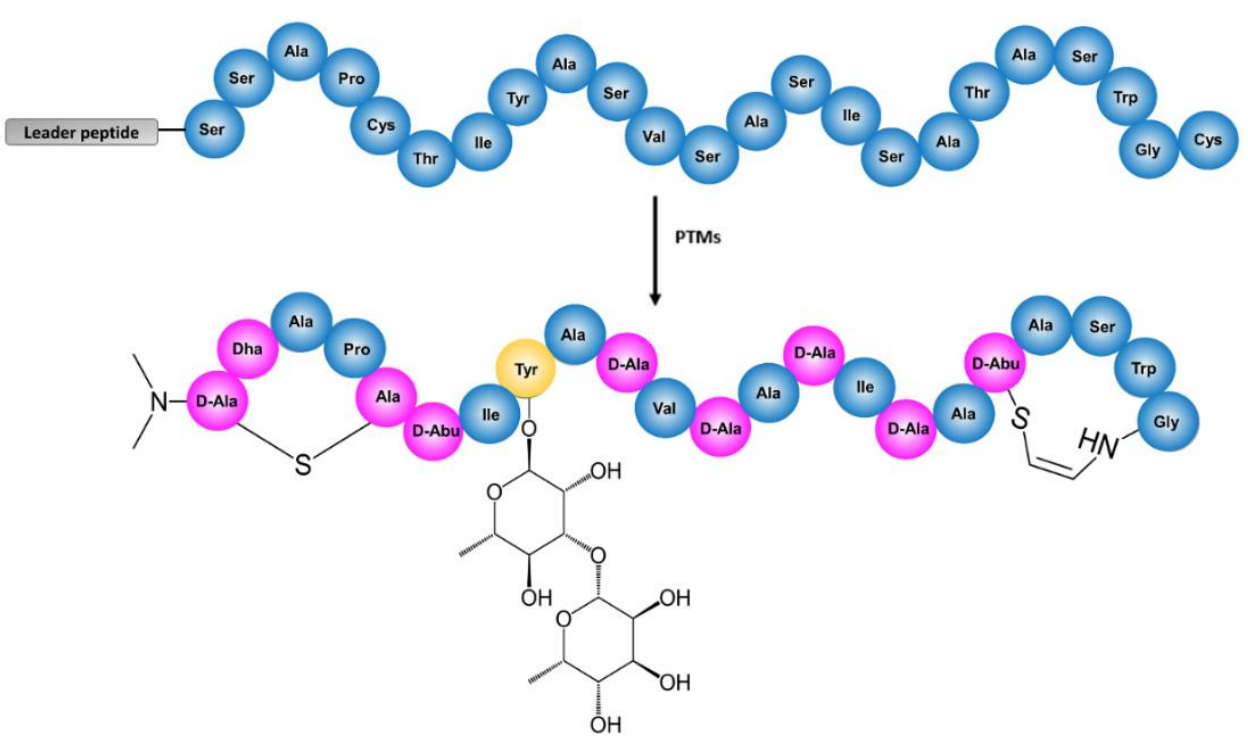

Figure 1. Structure of cacaoidin before (top) and after (bottom) the Post-Translational Modifications (PTMs). In the modified cacaoidin, absolute configuration for NMe2Ala(S)-1 is tentatively proposed as (2S) (equivalently, D-Ala). The amino acids modified by biosynthetic enzymes are colored in pink, the glycosylated tyrosine is colored in yellow, and the non-modified amino acids are colored in blue.

Shortly after the discovery of cacaoidin, $\mathrm{Xu}$ et al. [20] described the novel RiPP lexapeptide as the first member of the new class $\mathrm{V}$ lanthipeptide. Lexapeptide is structurally similar to cacaoidin since it contains an $\mathrm{N}$-terminal $\mathrm{N}, \mathrm{N}$-dimethylation, a C-terminal AviMeCys moiety and a D-Ala. According to this, and to the new RiPP classification established in a recent review [21], cacaoidin is the founding member of the class $\mathrm{V}$ lanthipeptides, firstly reported by our group as lanthidins.

In this work, we present the identification and analysis of the cacaoidin BGC from the genome analysis of Streptomyces cacaoi CA-170360, showing its distinct gene cluster organization. We show that the BGC proposed contains all the genes required for cacaoidin biosynthesis that was successfully produced via heterologous expression. To our knowledge, this is the first class V lanthipeptide to be heterologously produced. We also describe that the cacaoidin BGC is a species-specific trait within the genus Streptomyces and identify the presence of other class $V$ lanthipeptide pathways in public genome databases.

\section{Results}

2.1. Sequencing of Streptomyces Cacaoi CA-170360 Genome and Identification of Cacaoidin BGC

S. cacaoi CA-170360 genome sequence was obtained with a combination of de novo PacBio and Illumina approaches, yielding two contigs of 5,971,081 bp and 2,704,105 bp.

The genome sequence was analyzed with antiSMASH [6], BAGEL4 [22] and PRISM [23], but none of these tools could predict the BGC responsible for cacaoidin biosynthesis, suggesting that the discovery of novel bioactive NPs using genome mining is still a challenge [10].

The C-terminal sequence of cacaoidin (Thr-Ala-Ser-Trp-Gly-Cys) was used as the query in a tBLASTn using the whole genome sequence. A $162 \mathrm{bp}$ Open Reading Frame (ORF) was found to encode this sequence and helped to elucidate the final structure of 
the peptide [12]. Cacaoidin structural gene $c a 0 A$ encodes a 23-amino acid C-terminal core peptide (SSAPCTIYASVSASISATASWGC) following a predicted 30-amino acid N-terminal leader peptide (MGEVVEMVAGFDTYADVEELNQIAVGEAPE). Neither the leader nor the core peptide sequences showed high sequence similarity with any other lanthipeptide or linaridin (Figure S1).

Considering the structure of cacaoidin [12] (Figure 1), we identified a putative $30 \mathrm{~Kb}$ BGC (cao cluster) containing 27 ORFs whose proposed functions were assigned after BLAST analysis and HHpred secondary structure prediction (Figure 2, Table 1 and Table S2). Interestingly, no homologous genes of known dehydratases or cyclases commonly found in the four classes described for lanthipeptides nor in the class of linaridins could be identified in this region. Moreover, the similarity with the recently described lexapeptide BGC $(l x m)$ [20] contributed as well to assigning some of the functions of the cao ORFs.

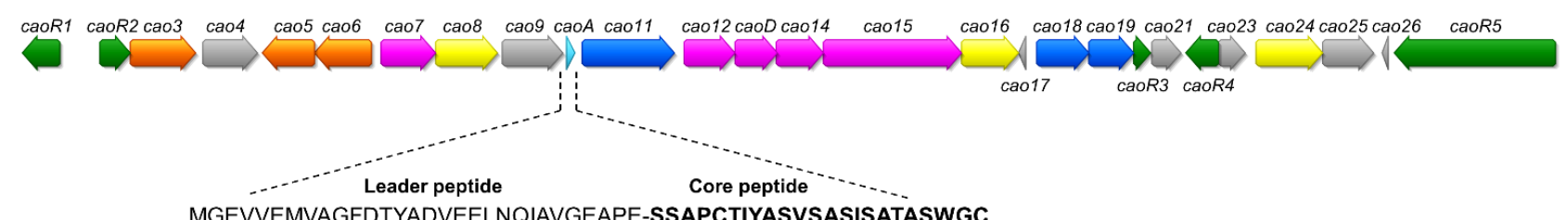

MGEVVEMVAGFDTYADVEELNQIAVGEAPE-SSAPCTIYASVSASISATASWGC

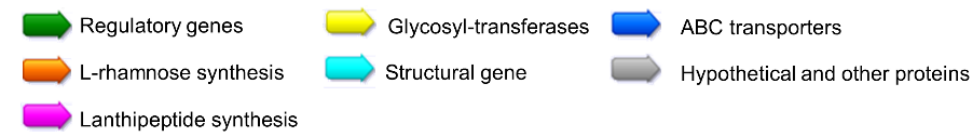

Figure 2. Schematic representation of the BGC of cacaoidin, where $c a \circ A$ codes for the precursor peptide. The sequences of the leader and core peptides of cacaoidin are shown.

Table 1. Closest BLAST homolog for each Open Reading Frame (ORF) in cacaoidin Biosythetic Gene Cluster (BGC).

\begin{tabular}{|c|c|c|c|c|}
\hline ORF & Closest BLAST Homolog & $\begin{array}{l}\text { \%Identity/ } \\
\text { Similarity }\end{array}$ & Conserved Domains & Possible Function \\
\hline caoR1 & $\begin{array}{l}\text { DNA-binding response regulator } \\
\text { [Streptomyces cacaoi subsp. cacaoi] }\end{array}$ & $100 / 100$ & $\begin{array}{c}\text { CitB (NarL/FixJ family, contains REC and } \\
\text { HTH domains); HTH_LUXR }\end{array}$ & Positive regulator \\
\hline caoR2 & $\begin{array}{l}\text { Helix-turn-helix domain-containing protein } \\
\text { [Streptomyces cacaoi] }\end{array}$ & $98.44 / 100$ & HTH_XRE superfamily & Negative regulator \\
\hline cao3 & $\begin{array}{l}\text { Hypothetical protein SCA03_05120 } \\
\text { [Streptomyces cacaoi subsp. cacaoi] }\end{array}$ & $97.81 / 100$ & RmlD_sub_bind & L-rhamnose synthesis \\
\hline cao4 & Methyltransferase [Streptomyces cacaoi] & $100 / 100$ & Methyltrans_2 (O-methyltransferase) & N-terminal dimethylation \\
\hline$c a 05$ & $\begin{array}{l}\text { GDP-mannose 4,6-dehydratase } \\
\text { [Streptomyces cacaoi }]\end{array}$ & $98.16 / 100$ & $\begin{array}{l}\text { dTDP_GD_SDR-e (dDTP-D-glucose } \\
\text { 4,6-dehydratase) }\end{array}$ & L-rhamnose biosynthesis \\
\hline cao6 & $\begin{array}{l}\text { MULTISPECIES: glucose-1-phosphate } \\
\text { thymidylyltransferase [Streptomyces] }\end{array}$ & $100 / 100$ & $\begin{array}{l}\text { RmlA_long (glucose-1-phosphate } \\
\text { thymidylyltransferase) }\end{array}$ & L-rhamnose biosynthesis \\
\hline cao7 & Hypothetical protein [Streptomyces cacaoi] & $100 / 100$ & HopA1 superfamily & Unknown \\
\hline$c a 08$ & $\begin{array}{l}\text { Glycosyltransferase family } 2 \text { protein } \\
\text { [Streptomyces sp. NRRL S-1868] }\end{array}$ & $99.48 / 100$ & Glycos_transf_2 & Glycosylation \\
\hline$c a 09$ & $\begin{array}{l}\text { Phosphotransferase } \\
\text { [Streptomyces sp. NRRL S-1868] }\end{array}$ & $100 / 100$ & PKc_like (protein kinase catalytic domain) & Unknown \\
\hline$c a o A$ & $\begin{array}{l}\text { Hypothetical protein SCA03_05190 } \\
\text { [Streptomyces cacaoi subsp. cacaoi] }\end{array}$ & $100 / 100$ & No putative conserved domains detected & Structural gene \\
\hline cao11 & $\begin{array}{l}\text { ABC transporter ATP-binding protein } \\
\text { [Streptomyces sp. NRRL F-5053] }\end{array}$ & $99.66 / 100$ & $\begin{array}{c}\text { MdlB (ABC-type multidrug transport } \\
\text { system, ATPase and permease component) }\end{array}$ & Cacaoidin biosynthesis \\
\hline cao12 & $\begin{array}{l}\text { MULTISPECIES: LLM class flavin-dependent } \\
\text { oxidoreductase [Streptomyces] }\end{array}$ & $100 / 100$ & SsuD (Flavin-dependent oxidoreductase) & Cacaoidin biosynthesis \\
\hline$c a o D$ & $\begin{array}{l}\text { Hypothetical protein SCA03_05220 } \\
\text { [Streptomyces cacaoi subsp. cacaoi] }\end{array}$ & $99.63 / 100$ & $\begin{array}{c}\text { PRK05579 (bifunctional } \\
\text { phosphopantothenoylcysteine decarboxy- } \\
\text { lase/phosphopantothenate synthase) }\end{array}$ & AviMeCys biosynthesis \\
\hline
\end{tabular}


Table 1. Cont.

\begin{tabular}{|c|c|c|c|c|}
\hline ORF & Closest BLAST Homolog & $\begin{array}{l}\text { \%Identity/ } \\
\text { Similarity }\end{array}$ & Conserved Domains & Possible Function \\
\hline cao14 & $\begin{array}{l}\text { MULTISPECIES: hypothetical protein } \\
\text { [unclassified Streptomyces] }\end{array}$ & $99.34 / 100$ & No putative conserved domains detected & Unknown \\
\hline cao15 & Hypothetical protein [Streptomyces sp. NHF165] & $99.42 / 100$ & PqqL (predicted Zn-dependent peptidase) & Leader peptide cleavage \\
\hline cao16 & $\begin{array}{l}\text { Glycosyltransferase family } 2 \text { protein } \\
\text { [Streptomyces cacaoi] }\end{array}$ & $100 / 100$ & Glycos_transf_2 & Glycosylation \\
\hline cao17 & Hypothetical protein [Streptomyces cacaoi] & $91.7 / 100$ & No putative conserved domains detected & Unknown \\
\hline cao18 & $\begin{array}{l}\text { ABC transporter ATP-binding protein } \\
\text { [Streptomyces sp. NRRL F-5053] }\end{array}$ & $100 / 100$ & $\begin{array}{l}\text { CcmA (ABC-type multidrug } \\
\text { transport system) }\end{array}$ & Cacaoidin biosynthesis \\
\hline cao19 & $\begin{array}{l}\text { MULTISPECIES: ABC transporter permease } \\
\text { [Streptomyces] }\end{array}$ & $99.65 / 100$ & $\begin{array}{l}\text { ABC2_membrane_3 (ABC-2 family } \\
\text { transporter protein) }\end{array}$ & Cacaoidin biosynthesis \\
\hline caoR3 & $\begin{array}{l}\text { Hypothetical protein SCA03_05280 } \\
\text { [Streptomyces cacaoi subsp. cacaoi] }\end{array}$ & $100 / 100$ & HTH_XRE domain & Negative regulator \\
\hline cao21 & $\begin{array}{c}\text { Hypothetical protein } \\
\text { [Streptomyces sp. NRRL S-1868] }\end{array}$ & $100 / 100$ & No putative conserved domains detected & Unknown \\
\hline caoR4 & $\begin{array}{c}\text { TetR/AcrR family transcriptional regulator } \\
\text { [Streptomyces cacaoi] }\end{array}$ & $100 / 100$ & $\begin{array}{l}\text { AcrR (DNA-binding } \\
\text { transcriptional regulator) }\end{array}$ & Negative regulator \\
\hline cao23 & $\begin{array}{l}\text { Hypothetical protein SCA03_05310 } \\
\text { [Streptomyces cacaoi subsp. cacaoi] }\end{array}$ & $99.39 / 100$ & SRPBCC superfamily & Unknown \\
\hline cao 24 & $\begin{array}{l}\text { MULTISPECIES: glycosyltransferase family } 4 \\
\text { protein [Streptomyces] }\end{array}$ & $100 / 100$ & GT4_AmsD-like & Glycosylation \\
\hline cao25 & Hypothetical protein [Streptomyces cacaoi] & $99.06 / 100$ & No putative conserved domains detected & Unknown \\
\hline cao26 & No homologues found & - & - & Unknown \\
\hline caoR5 & $\begin{array}{l}\text { Tetratricopeptide repeat protein } \\
\text { [Streptomyces sp. NRRL S-1868] }\end{array}$ & $99.31 / 100$ & BTAD (Bacterial Transcriptional Activation) & Positive regulator \\
\hline
\end{tabular}

The cao BGC encodes a putative cypemycin decarboxylase CypD homologue (CaoD) containing a conserved phosphopantothenoylcysteine (PPC) synthetase/decarboxylase domain $[17,24]$. CaoD shares a $54.8 \%$ similarity with LxmD from the lexapeptide BGC, which is involved in the decarboxylation of $\mathrm{C}$-terminal Cys residue during the formation of the AviMeCys moiety [20], a fact that supports the same role for CaoD.

The formation of lanthionine rings is accomplished by different dehydratases and cyclases depending on the lanthipeptide class [15], but no homologues were found in the cao cluster. In the lexapeptide biosynthesis, a three-component lanthionine synthetase formed by the standalone monofunctional proteins LxmK, LxmX and LxmY is responsible for the Ser/Thr dehydration and cyclization to form Dha and Dhb, respectively, and the installation of the lanthionine ring [20]. These proteins present a certain degree of homology with some proteins encoded in the cao cluster: LxmK shows a 49.6\% similarity with Cao9, LxmX has a $40.5 \%$ similarity with Cao14 and LxmY shows a $44.9 \%$ similarity with Cao7. Interestingly, LxmY and Cao7 contain a HopA1 conserved domain (PFAM17914) that has been described in the HopA1 effector protein from Pseudomonas syringae [25].

According to these data, Cao7-9-14 would also work as a three-component lanthionine synthetase: the putative kinase Cao9 would phosphorylate Ser/Thr residues on the CaoA precursor peptide, Cao14 would remove the phosphate groups and generate dehydroamino acids, and Cao7 would catalyze a Michael-type addition of the Cys5 thiol group to the $\beta$-carbon of Dha1, generating the lanthionine bridge, and likely the addition of previously decarboxylated-Cys23 by CaoD to Dhb18, forming AviMeCys.

The N-terminal Ala dimethylation of cypemycin, the prototypical member of linaridins [18], is carried out by the S-adenosylmethionine (SAM)-dependent methyltransferase CypM $[14,19]$ and shows homology with the methyltransferase LxmM that catalyzes the lexapeptide N-terminal dimethylation [20]. The cao cluster encodes Cao4, a putative Omethyltransferase containing the conserved Methyltransf_2 domain. However, despite the low homology found between Cao4 and LxmM (26.9\% similarity), both proteins share the AdoMet_MTases superfamily domain, characteristic of the SAM-dependent methyltransferases. No other CypM or LxmM homologues have been found in the genome of strain CA-170360. Cao4 shows very low homology with two types of LanS O-methyltransferases 
described in class I lanthipeptide clusters from actinobacteria: $\mathrm{LanS}_{\mathrm{A}}$ [26] and $\mathrm{LanS}_{\mathrm{B}}$ [27]. Thus, its role in the $\mathrm{N}, \mathrm{N}$-methylation remains unclear and is currently under study.

D-Amino acids provide a wide variety of properties to lanthipeptides [28] and are introduced by modifying the genetically encoded L-Ser and L-Thr into Dha and Dhb, which are subjected to a diastereoselective hydrogenation to finally incorporate D-Ala and $\mathrm{D}-\mathrm{Abu}$, respectively $[15,27]$. This reaction is carried out by dehydrogenases generically called LanJ [15], which are divided into zinc-dependent dehydrogenases $\left(\operatorname{LanJ}_{\mathrm{A}}\right)$, which can only hydrogenate Dha, and flavin-dependent dehydrogenases $\left(\operatorname{LanJ}_{\mathrm{B}}\right)$ able to reduce both Dha and Dhb $[29,30]$. Xu et al. [20] reported LxmJ as the first example of the LanJ $\mathrm{C}_{\mathrm{C}}$ class in lanthipeptides. LxmJ is a novel $\mathrm{F}_{420} \mathrm{H}_{2}$-dependent reductase that catalyzes the stereospecific reduction of Dha28 to D-Ala28 in the mature lexapeptide. Within the cao BGC, Cao12 shows a 58,6\% similarity with LxmJ, and both proteins contain a $\mathrm{F}_{420} \mathrm{H}_{2-}$ dependent oxidoreductase (MSMEG_4879 family) conserved domain. This suggests that Cao12 could be also involved in the incorporation of D-amino acids (four D-Ala and one $\mathrm{D}-\mathrm{Abu}$ ) in the cacaoidin structure. It has been demonstrated that $\mathrm{LxmJ}$ is unable to reduce Dhb when a S28T point mutation is introduced in LxmA. However, as it was shown for $\mathrm{BsjJ}_{\mathrm{B}}$ in bicereucin biosynthesis [30], the reduction may be dependent of the position and/or sequence, so the incorporation of D-Abu in cacaoidin could also be performed by Cao12.

The disaccharide moiety $\beta$-6-deoxygulopyranosyl-( $1 \rightarrow 3)$ - $\alpha$-rhamnopyranoside of cacaoidin has not been previously reported [12]. Three of the four proteins required for the synthesis of $\alpha$-L-rhamnose ( $r m l A, r m l B$ and $r m l D$ ) (Figure S2) are encoded in the cao cluster. Nevertheless, a BLAST search of RmlC against CA-170360 whole genome sequence also shows the presence of a $r m l C$ gene and additional $r m l A, r m l B$ and $r m l D$ homologous genes outside the cacaoidin cluster. Indeed, it has been reported that the $\mathrm{rml}$ genes do not have to be necessarily clustered [31]. Bleomycin, tallysomycin and zorbamycin incorporate NDP-L-gulose or NDP-6-deoxy-L-gulose to their structures, and their biosynthesis was used as reference to look for the presence of similar proteins being encoded in our genome (Figure S3) [32]. Despite no homologues being found in the cacaoidin BGC, we identified some protein homologues in the genome. However, as none of them were clustered, no conclusions could be made for the $\beta$-L-6-deoxy-gulose biosynthesis.

As one of the unusual structural features of cacaoidin, the disaccharide is O-linked to the aromatic ring of the tyrosine residue [12]. While serine, threonine or hydroxyproline O-glycosylation have been reported in many natural glycopeptides [33], the O-glycosylation of tyrosine in a natural product has been only reported in the mannopeptimycins, in a reaction catalyzed by the mannosyltransferases MppH and MppI [34]. The cao cluster lacks MppH/I homologues, but contains three glycosyltransferases (GTs) (Cao8, Cao16 and Cao24). Cao8 and Cao16 belong to the family GT- 2 and show $42 \%$ identity ( $54 \%$ similarity) and $43 \%$ identity (52\% similarity), respectively, with an UDP-Glc:alpha-D-GlcNAc-diphosphoundecaprenol beta1,3-glucosyltransferase $\mathrm{WfgD}$, which catalyzes the addition of Glc, the second sugar moiety of the O152-antigen repeating unit, to GlcNAc-pyrophosphate-undecaprenol [35]. In contrast, Cao24 belongs to the family GT-4 that has a GT4_GtfA-like domain and a conserved RfaB domain, involved in the cell wall and membrane biosynthesis [36].

Despite the presence of three GTs in the cacaoidin BGC, only two sugar moieties in the form of disaccharide are detected in the structure. The three GTs could work in a cooperative manner to achieve effective glycosylation, as it has already been proposed [37-39]. Based on the "inverting" or "retaining" behavior of the glycosyltransferases, we propose that both Cao8 and Cao16, belonging to the "inverting" GT-2 family, might work cooperatively to attach the $\alpha$-L-rhamnose unit, while Cao24, belonging to the "retaining" GT-4 family, would incorporate the $\beta$-L-6-deoxygulose unit [12].

In addition to cacaoidin, only two glycosylated RiPPs produced by bacteria have been described: the group of glycocins, whose sugar moieties are linked to Cys, Ser or Thr residues by S-glycosyltransferases [40], and the class III lanthipeptide NAI-112, which carries a 6-deoxyhexose moiety N-linked to a tryptophan residue by the ApIG GT [41]. 
However, low homologies were found between these enzymes and the GTs present in the cacaoidin cluster. Further studies will clarify the role of each GT in cacaoidin biosynthesis.

Processing of leader peptide is another key step in the post-translational modification of RiPPs [15,42-46]. In the cacaoidin cluster, Cao15 encodes a putative Zn-dependent peptidase belonging to the M16 peptidase family that may be involved in the leader peptide processing. Peptidases LxmP1 and LxmP2 from lexapeptide BGC also belong to the M16 peptidase family and share $25.4 \%$ and $30.4 \%$ similarity with Cao15, respectively. LxmP1 has an inactive M16 peptidase domain and LxmP2 an active domain. It has been suggested that LxmP1 and LxmP2 may form a heterodimer to remove the leader peptide from LxmA [20]. Since Cao15 harbors both the active and the inactive M16 peptidase domains, we propose Cao15 as the cacaoidin leader peptidase.

Furthermore, three ABC transporters were found in the cao pathway (Cao11, Cao18 and Cao19) that might be responsible for the export and self-resistance of cacaoidin, as it has been proposed in other lanthipeptides $[47,48]$.

Gene expression in the cacaoidin cluster seems to be under the control of five different transcriptional regulators: one LuxR (CaoR1), two HTH-type XRE (CaoR2 and CaoR3), one TetR (CaoR4) and one SARP (CaoR5). XRE and TetR have been described as transcriptional repressors $[49,50]$ while LuxR and SARP have been designated as transcriptional activators [51,52]. Further studies will clarify their role in the production of the antibiotic.

The remaining five genes identified in the cao cluster (cao17, cao21, cao23, cao25 and cao26) do not have any defined functions. Cao23 belongs to the SRPBCC (START/RHO_alpha_C/ PITP/Bet_v1/CoxG/CalC) superfamily of proteins [53,54]. This superfamily contains aromatase/cyclase (ARO/CYC) domains, such as those described for tetracenomycin [55] and Smu.440 [56]. However, we cannot still propose a function for Cao23.

The HHpred analysis of each ORF was also used for the detection of RiPP precursor peptide Recognition Elements (RREs) [57]. These RRE are structurally similar PqqDrelated conserved precursor peptide-binding domains present in the majority of known prokaryotic RiPP modifying enzymes and are usually responsible for the leader peptide recognition [57-59]. However, no RREs were found, suggesting the possibility of alternative leader peptide recognition domains that are unrelated to the already known RREs [57]. As homology detection algorithms become more accurate and more sequences become available, additional RREs will be found.

\subsection{Cloning and Heterologous Expression of Cacaoidin BGC}

The strain S. cacaoi CA-170360 is reluctant to genetic manipulation, limiting the obtention of knockdown mutants to confirm the involvement of the cao gene cluster in the biosynthesis of cacaoidin. To confirm that the genes included in the cao cluster were sufficient for biosynthesis of the antibiotic, we cloned and heterologously expressed the cacaoidin BGC in the genetically amenable host Streptomyces albus J1074 [60].

We followed the CATCH method [61] to clone a $40 \mathrm{~Kb}$ region containing the cao BGC into the pCAP01 vector [62], yielding pCAO. pCAO was introduced into NEB-10-beta E. coli ET12567 cells via electroporation. A triparental conjugation [63] was carried out between E. coli ET12567/pCAO, E. coli ET12567/pUB307 and S. albus J1074 spores [60]. The transconjugants were genetically verified via PCR amplification of the genes cao4, cao8 and cao15 using specific primers described in Table S1. Five positive transconjugants, alongside the negative control (S. albus J1074/pCAP01) and the wild-type strain CA-170360, were grown in R2YE, MPG, FR23, YEME and KM4 for 14 days at $28{ }^{\circ} \mathrm{C}$ to confirm the production of the targeted antibiotic. After acetone extraction of the cultures, organic solvent was evaporated, and the aqueous extracts containing 20\% DMSO were analyzed via LC-HRESIMS(+)-TOF and MS/MS.

The analysis of the extracts from pCAO transconjugants confirmed the presence of peaks at $3.35 \mathrm{~min}$, coincident with the retention time of elution of cacaoidin in the wild-type strain and purified cacaoidin standards, only in R2YE medium. The perfect correlation between the UV spectrum, exact mass, isotopic distribution and MS/MS fragmentation 
patterns of cacaoidin standards and the components isolated from S. albus J1074/pCAO, undoubtedly demonstrated that they correspond to cacaoidin (Figures 3 and 4). We quantified the yield of cacaoidin production in both the heterologous and the wild-type strains. S. albus J1074 has been largely used as heterologous host to produce a broad range of compound classes with improved yields [64]. However, the cacaoidin production yield of $0.14 \mathrm{mg} / \mathrm{L}$ in the heterologous strain grown in R2YE was reduced approximately 4-fold with respect to the $0.57 \mathrm{mg} / \mathrm{L}$ obtained in the wild-type strain CA-170360 in the same medium and fermentation format $(10 \mathrm{~mL})$. These results clearly confirm that the cao BGC cloned in pCAO contains all the genes required to ensure the biosynthesis of cacaoidin, although the production yield is dependent on the strain used.

a
b
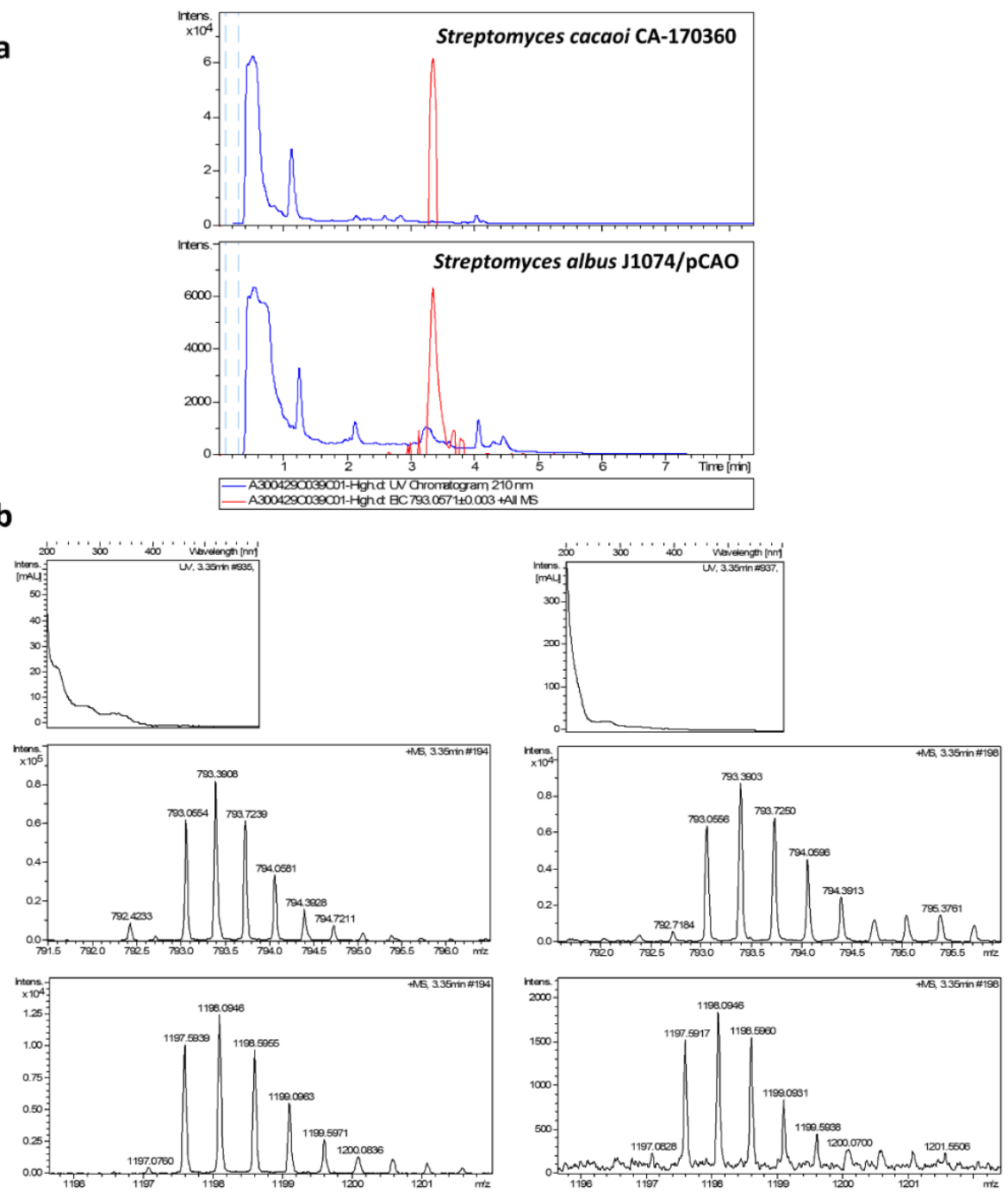

Figure 3. (a) Chromatograms of UV absorbance at $210 \mathrm{~nm}$ (blue trace) and extracted ion $m / z=793.0571 \pm 0.003, \mathrm{C}_{107} \mathrm{H}_{162} \mathrm{~N}_{24} \mathrm{O}_{32} \mathrm{~S}_{2}+\mathrm{NH}_{4}{ }^{+}+2 \mathrm{H}^{+}$of cacaoidin (MDN-0207) from original producing strain Streptomyces cacaoi CA-170360 and the heterologous producing strain Streptomyces albus J1074 /pCAO. (b) Experimental UV and positive mass spectra from $\mathrm{C}_{107} \mathrm{H}_{162} \mathrm{~N}_{24} \mathrm{O}_{32} \mathrm{~S}_{2}+\mathrm{NH}_{4}{ }^{+}$ $+2 \mathrm{H}^{+}$(calculated value: 793.0571) (top) and $\mathrm{C}_{107} \mathrm{H}_{162} \mathrm{~N}_{24} \mathrm{O}_{32} \mathrm{~S}_{2}+2 \mathrm{NH}_{4}{ }^{+}$(calculated value: 1197.5952) (bottom) adducts from heterologous producing strain (right) and from original producing strain CA-170360 (left). 
a
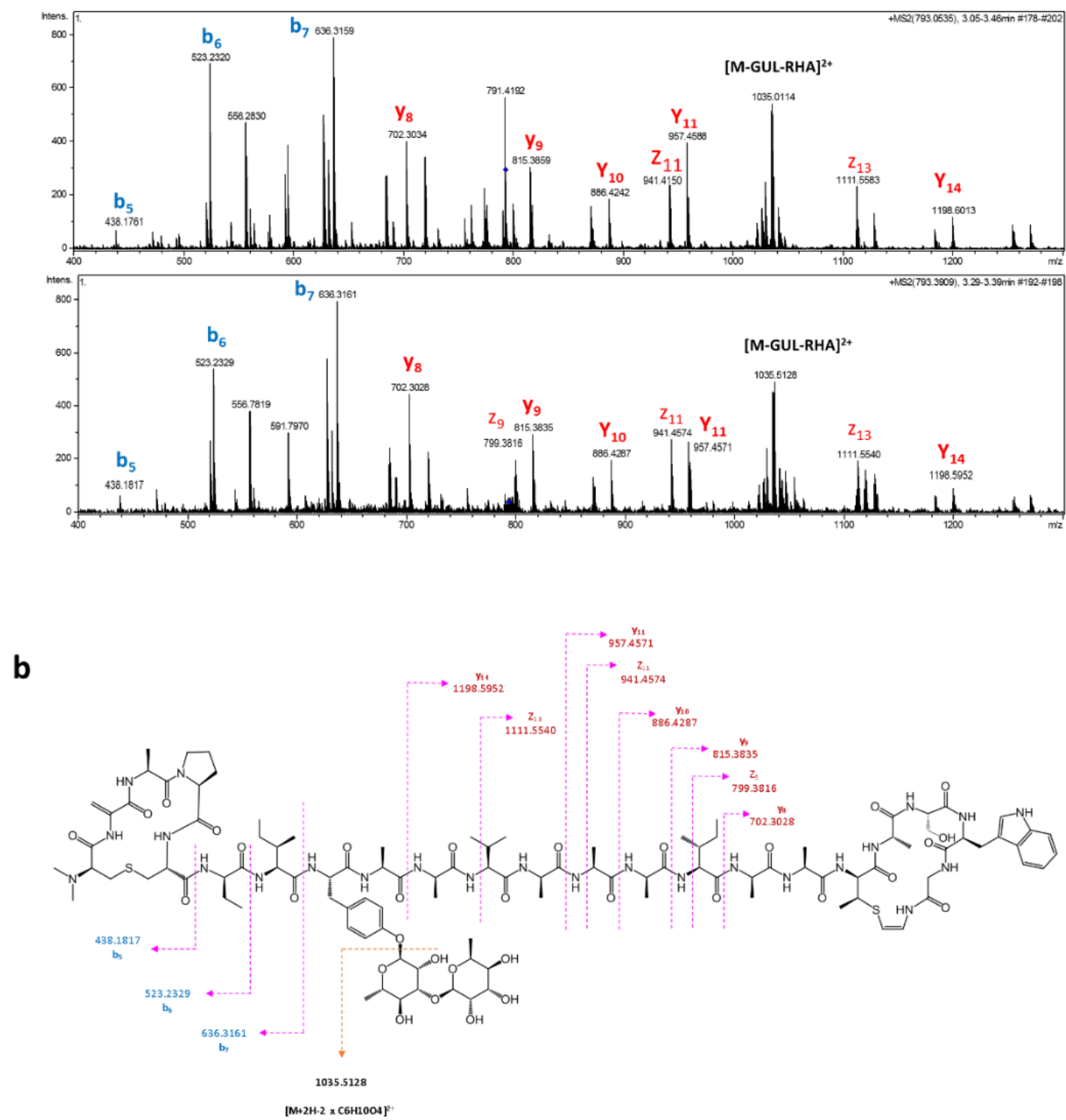

Figure 4. (a) Tandem MS/MS fragmentation pattern of $\mathrm{C}_{107} \mathrm{H}_{162} \mathrm{~N}_{24} \mathrm{O}_{32} \mathrm{~S}_{2}+\mathrm{NH}_{4}{ }^{+}+2 \mathrm{H}^{+}$from strain CA-170360 (top) and S. albus $\mathrm{J} 1074 / \mathrm{pCAO}$ (bottom). Annotation of b-, $\mathrm{y}$ - and z-ions is shown. (b) Key MS/MS fragmentation of $[\mathrm{M}+2 \mathrm{H}+$ $\left.\mathrm{NH}_{4}\right]^{3+}$ ion.

\subsection{Additional Lanthidin Clusters in Public Databases}

To study if more lanthidin-encoding clusters can be found within actinomycetes, a BLAST search against the NCBI whole genome shotgun sequences database was performed, and sequences with a high degree of homology to cacaoidin BGC were only found in five Streptomyces strains, namely Streptomyces cacaoi subsp. cacaoi NRRL B-1220 (MUBL01000486), Streptomyces sp. NRRL F-5053 (JOHT01000009), Streptomyces sp. NRRL S-1868 (JOGD01000003), Streptomyces cacaoi subsp. cacaoi NBRC 12748 (BJMM01000002.1) and Streptomyces cacaoi subsp. cacaoi OABC16 (VSKT010000024) (Figure 5, Table S3).

An alignment of the precursor peptide of the cacaoidin in all homologous clusters showed that no variations in the protein sequence were found. No other cacaoidin-derived peptides or pathways were found in the databases, indicating that the cacaoidin BGC is very conserved. A phylogenetic tree generated using the neighbor-joining method and corrected with the Tamura 3-parameter algorithm $[65,66]$ showed the close relatedness of strain Streptomyces cacaoi CA-170360 with the other five strains that contain the cao cluster, 
which was highly supported by the bootstrap values and contained two sequences of the type species of S.cacaoi subsp cacaoi NRRL B-1220 and NBRC 12748, as well as the strain of the same subspecies, OABC16 (Figure S4). Moreover, when the 16S rDNA sequences of the unclassified strains Streptomyces sp. NRRL F-5053 and Streptomyces sp. NRRL S-1868 were analyzed in EzBiocloud, a 100\% sequence similarity was confirmed with Streptomyces cacaoi subsp. cacaoi NRRL B-1220, indicating that the cacaoidin BGC is so far limited to this specific clade of highly related strains associated to the subspecies $S$. cacaoi subsp. cacaoi, with no identifiable orthologs in other Streptomyces species. We extended the study to other members of the subspecies S.cacaoi subsp. asoensis that are clustered in a very distant phylogenetic branch from S.cacaoi subsp. cacaoi (Figure S4). We could confirm that none of the three whole genome sequences available in NCBI from strains of $S$. cacaoi subsp. asoensis contained any region with homology with the cao cluster, supporting that the cao BGC is a characteristic trait of the subspecies $S$. cacaoi subsp cacaoi. Several genome comparative studies have found other species-specific BGCs in some species of Streptomyces, reflecting that chemical novelty can be found at the species level and that the analysis of the genomes of closely related strains constitutes a promising approach for the identification of novel BGCs $[67,68]$. The occurrence of these species-specific BGCs may suggest that they were acquired recently via horizontal gene transfer as it has been shown for other pathways [69].

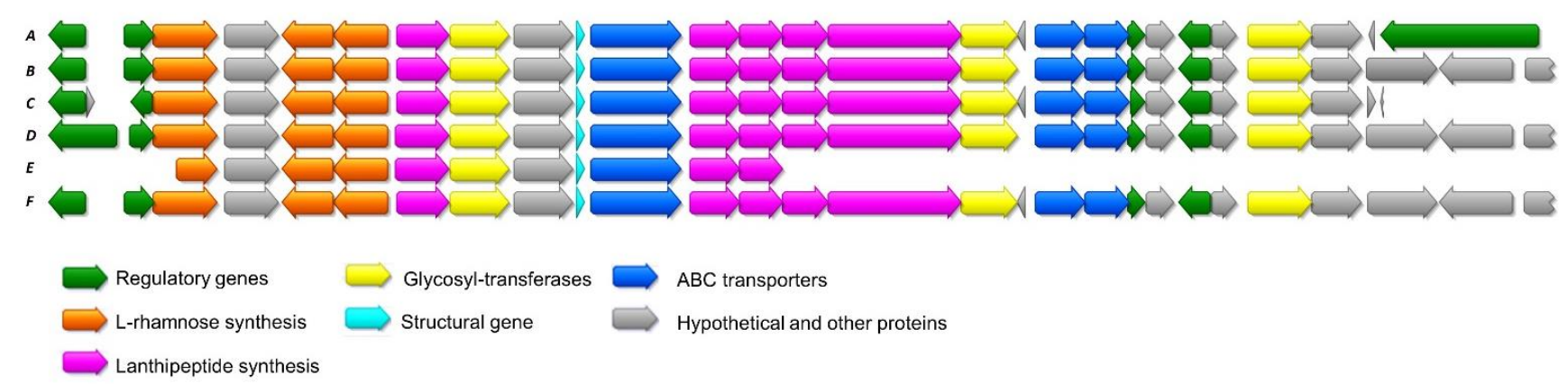

Figure 5. Schematic representation of the alignment of cacaoidin BGC from Streptomyces cacaoi CA-170360 and the highly homologous clusters found in NCBI. All of them belong as well to strains of S. cacaoi. A: S. cacaoi CA-170360; B: S. cacaoi subsp. cacaoi NBRC 12748; C: Streptomyces sp. NRRL S-1868; D: Streptomyces sp. NRRL F-5053; E: S. cacaoi subsp. cacaoi NRRL B-1220; F: S. cacaoi subsp. cacaoi OABC16.

Nevertheless, the analysis of below-threshold scores of CaoA BLAST results, together with the search of HopA1 domain-containing proteins similar to Cao7, allowed us to find some additional pathways that could encode new lanthidins (Figure 6). The alignment of the hypothetical precursor peptides shows the presence of some conserved residues that possibly could be involved in the leader peptide recognition by biosynthetic enzymes (Figure 7). In addition, the analysis of the ORFs present in all these clusters shows that all of them share a HopA1 domain-containing protein, a $\mathrm{F}_{420} \mathrm{H}_{2}$-dependent oxidoreductase, a CypD-related protein, a Zn-dependent or S9 peptidase and a putative phosphotransferase (Figure 6). Most of these clusters also contain an O-methyltransferase. These preliminary data suggest the existence of a minimal set of genes required to ensure the core structural features of a lanthidin and a broader distribution of potential BGCs encoding new lanthidins. These putative lanthidin BGCs are mainly present in Streptomyces strains, but also in two Actinoplanes species and another undefined member of the class Actinobacteria. To date, only the class V lanthipeptides cacaoidin and lexapeptide BGCs have been described. Additional members of the proposed new RiPP family will need to be isolated to confirm this hypothesis and whether class $\mathrm{V}$ lanthipeptides are only present in Actinobacteria (Figure 7). 


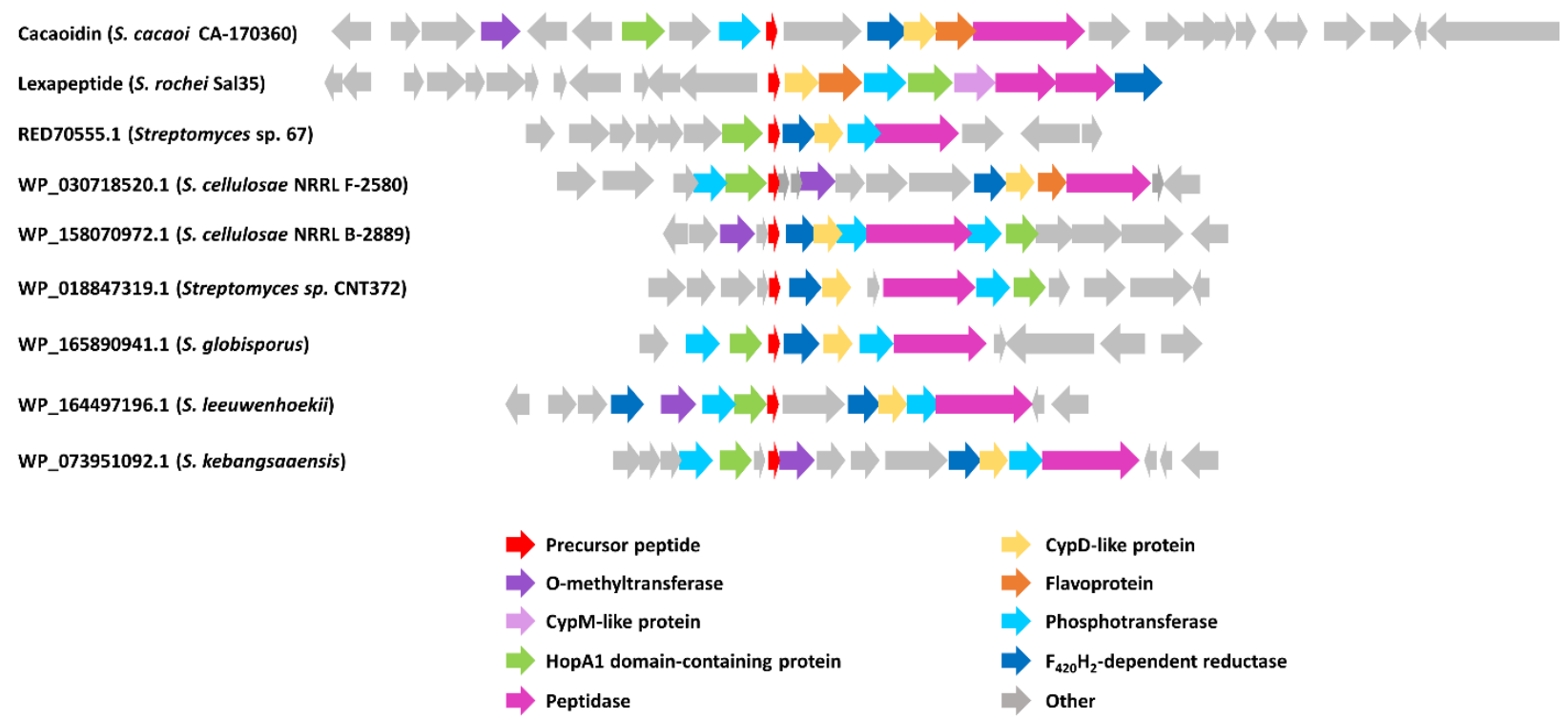

Figure 6. Schematic representation of cacaoidin BGC and the BGC of lexapeptide and other putative lanthidins (class V lanthipeptides). Accession numbers of each putative structural peptide are indicated.
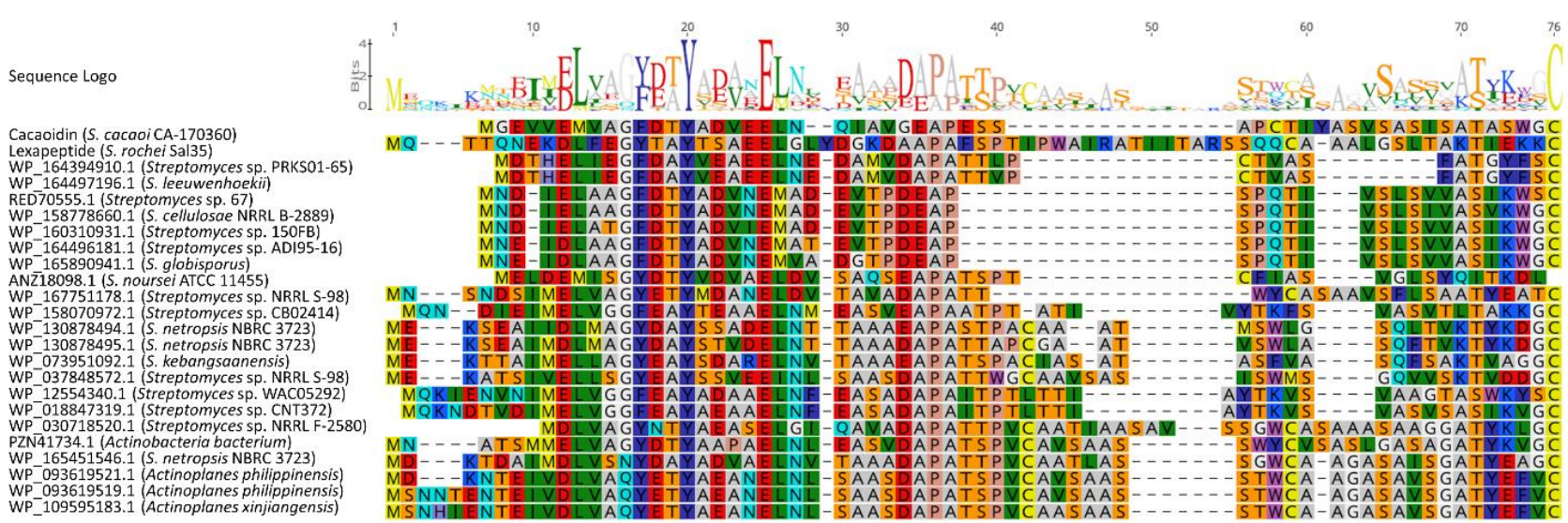

Figure 7. Alignment of cacaoidin and putative lanthidins (class V lanthipeptides) precursor peptides (accession numbers are shown). Sequence logo shows conserved residues.

\section{Materials and Methods}

\subsection{Strains and Plasmids}

The strain Streptomyces cacaoi CA-170360, from Fundación MEDINA's Culture Collection, was isolated from the rhizosphere of Brownanthus corallinus, in the region of Namaqualand (South Africa). Electrocompetent NEB 10- $\beta$ E. coli (New England BioLabs, Ipswich, MA, USA), E. coli ET12567 (LGC Standards, Manchester, NH, USA) and E. coli ET12567/pUB307 (kindly provided by José Antonio Salas) were used for plasmid transformation and intergeneric triparental conjugation. Streptomyces albus J1074 [60], also kindly provided by José Antonio Salas, was employed as the heterologous expression host.

Vector pCAP01, a yeast-E. coli-actinobacteria shuttle vector that can integrate cloned gene clusters into the genome of heterologous actinobacteria hosts due to its site-specific $\varphi \mathrm{C} 31$ integrase [70], was used for the cloning of the cacaoidin BGC and was a gift from Bradley Moore (Addgene plasmid \#59981; http:/ /n2t.net/addgene:59981 (accessed on 12 January 2021); RRID: Addgene_59981). 


\subsection{DNA Extraction and Genome Mining}

Genomic DNA of the strain Streptomyces cacaoi CA-170360 was extracted and purified as Kieser et al. described [63] from cultures on ATCC-2 liquid medium (soluble starch $20 \mathrm{~g} / \mathrm{L}$, glucose $10 \mathrm{~g} / \mathrm{L}$, NZ Amine Type E $5 \mathrm{~g} / \mathrm{L}$, meat extract $3 \mathrm{~g} / \mathrm{L}$, peptone $5 \mathrm{~g} / \mathrm{L}$, yeast extract $5 \mathrm{~g} / \mathrm{L}$, calcium carbonate $1 \mathrm{~g} / \mathrm{L}, \mathrm{pH}$ 7) grown on an orbital shaker at $28^{\circ} \mathrm{C}, 220 \mathrm{rpm}$ and $70 \%$ relative humidity.

The genome of CA-170360 was fully sequenced de novo, assembled and annotated by Macrogen (Seoul, Korea; http:/ / www.macrogen.com / (accessed on 12 January 2021)), using a combined strategy of Illumina HiSeq 2500 and PacBio RSII platforms. The PacBio long-reads were assembled with Canu (v1.7) [71]. After assembly, Illumina reads were applied for accurate genome sequence using Pilon (v1.21) [72]. To validate the accuracy of the assembly, Illumina reads were mapped to the assembly result. After mapping, the consensus sequence was generated.

\subsection{Identification of Cacaoidin BGC}

The sequence of the CA-170360 genome was analyzed with antiSMASH v4.2.0 [6], BAGEL4 [22], PRISM [23] and RiPPMiner [73] in order to find cacaoidin BGC. BLAST (Basic Local Alignment Search Tool) [74] and HHpred based on profile hidden Markov model (HMM) comparisons [75] were also employed to predict the function of each gene in the biosynthesis of the lanthipeptide. The cao BGC sequence is available in the National Center for Biotechnology Information (NCBI) database under accession GenBank number MT210103.

\subsection{Cas-9 Assisted Targeting of CHromosome (CATCH) Cloning of Cacaoidin BGC}

Cloning of cacaoidin BGC was performed via CATCH. The CRISPR-Cas9 endonuclease is guided by an RNA template to cleave specific DNA targets enabling larger BGC to be isolated than other techniques such as transformation-associated recombination or single-strand overlapping annealing [61].

The CATCH cloning was performed as Jian and Zhu described [76]. First, we designed the $20 \mathrm{nt}$ targeted sequences flanking the BGC with the CRISPy-web tool (http:/ / crispy. secondarymetabolites.org / (accessed on 12 January 2021)). This computational tool design guides RNAs close to a PAM (Protospacer-Adjacent Motif) sequence 'NGG' [77] and we performed an overlapping PCR with the Q5 High-Fidelity polymerase from New England BioLabs (Ipswich, MA, USA), and used one specific primer within the $20 \mathrm{nt}$ targeted sequence (X-sgRNA-P) and two universal primers (sgRNA-F and sgRNA-R). We obtained the sgRNA using the HiScribe T7 Quick Yield RNA synthesis kit (New England Biolabs). We also performed a PCR using the PCAP01 vector as backbone with primers containing a $20 \mathrm{nt}$ sequence that anneals to the template plasmid and a $30 \mathrm{nt}$ overhang overlapping with both ends of the BGC, and this PCR product was treated with DpnI (New England BioLabs) to cleave the template plasmid. Primers used for CATCH cloning are described in Table S1.

S. cacaoi CA-170360 was cultured in ATCC-2 for 2 days on an orbital shaker at $28^{\circ} \mathrm{C}$, $220 \mathrm{rpm}$ and $70 \%$ relative humidity and the bacterial cells were embedded in low-melting agarose plugs to make the subsequent in-gel digestion with Cas9 more feasible. The plugs were treated with lysozyme, proteinase $\mathrm{K}$ and washing buffers, and the in-gel Cas 9 digestion was performed by mixing two agarose plugs, a cleavage buffer $(100 \mathrm{mM}$ HEPES $\mathrm{pH}$ 7.5, $750 \mathrm{mM} \mathrm{KCl}, 0.5 \mathrm{mM}$ EDTA pH 8, $50 \mathrm{mM} \mathrm{MgCl2}$, DEPC-treated water), the sgRNAs and the Cas9 nuclease from S. pyogenes (New England BioLabs) and incubating at $37^{\circ} \mathrm{C}$ for $2 \mathrm{~h}$. After this time the agarose plugs were melted and digested with gelase and the cleavaged DNA was precipitated with ethanol and resuspended in DNase-free water.

The Cas9-digested DNA was cloned into the vector pCAP01 via Gibson Assembly using a $2 \times$ Gibson Assembly Master Mix (New England BioLabs) and incubating at $50{ }^{\circ} \mathrm{C}$ for $1 \mathrm{~h}$. After the ligation, this Gibson product (pCAO) was transformed into electrocompetent NEB-10-beta E. coli cells. These cells were incubated in $1 \mathrm{~mL}$ LB broth 
Miller (Sigma, St. Louis, MO, USA) $\left(37^{\circ} \mathrm{C}, 250 \mathrm{rpm}\right)$ without antibiotics and plated on Difco LB agar Lennox $\left(37^{\circ} \mathrm{C}\right.$, overnight, static) containing kanamycin $(50 \mu \mathrm{g} / \mathrm{mL})$.

The plasmids obtained from the selected colonies on these LB agar plates were validated by performing two independent double digestions with HindIII/NdeI and $\mathrm{XbaI} /$ EcoRV restriction endonucleases from New England BioLabs, which gave two different fragmentation patterns that allowed us to identify the correct pCAO construction.

\subsection{Heterologous Expression of Cacaoidin BGC}

Construction pCAO was introduced into Streptomyces albus J1074 host via triparental conjugation [61]. First, E. coli ET12567 cells were electroporated with $50 \mathrm{ng}$ of the construction in a total volume of $1 \mu \mathrm{L}$ and plated on selective LB agar plates with $50 \mu \mathrm{g} / \mathrm{mL}$ kanamycin. E. coli ET12567/pUB307 cells were also streaked on selective LB agar plates with $50 \mu \mathrm{g} / \mathrm{mL}$ kanamycin and $25 \mu \mathrm{g} / \mathrm{mL}$ chloramphenicol, which were incubated at $37^{\circ} \mathrm{C}$ overnight. E. coli ET12567/pCAO and ET12567/pUB307 cells were collected at an $\mathrm{OD}_{600}$ of 0.4-0.6, washed three times with LB liquid medium without antibiotics supplementation, suspended with $100 \mu \mathrm{L}$ LB liquid medium and mixed with $50 \mu \mathrm{L}$ of previously activated spores (incubated at $50{ }^{\circ} \mathrm{C}$ for $10 \mathrm{~min}$ ) of $\mathrm{S}$. albus $\mathrm{J} 1074$. These mixtures were plated onto MA plates and incubated at $28^{\circ} \mathrm{C}$ overnight (around $16 \mathrm{~h}$ ). On the next day, the conjugation MA plates were overlayed with $1.5 \mathrm{~mL}$ of sterilized Milli-Q water containing $50 \mu \mathrm{g} / \mathrm{mL}$ kanamycin and $25 \mu \mathrm{g} / \mathrm{mL}$ nalidixic acid. These plates were incubated again at $28{ }^{\circ} \mathrm{C}$ for 3-5 days to let the exconjugants grow. After this time of incubation, five exconjugants from each Streptomyces heterologous host were picked and spread out on MA plates containing nalidixic acid $(25 \mu \mathrm{g} / \mathrm{mL})$ and kanamycin $(50 \mu \mathrm{g} / \mathrm{mL})$.

Five plugs from these MA plates of the recombinant strain S. albus J1074/pCAO were used to seed $10 \mathrm{~mL}$ ATCC-2 tubes, which were incubated at $28^{\circ} \mathrm{C}$ for $2-3$ days, $220 \mathrm{rpm}$ and $70 \%$ humidity, and these cultures, together with the corresponding negative controls harboring empty pCAP01 vector, were used to inoculate $10 \mathrm{~mL}$ of MPG $(10 \mathrm{~g} / \mathrm{L}$ glucose, $20 \mathrm{~g} / \mathrm{L}$ Millet meal, $20 \mathrm{~g} / \mathrm{L}$ MOPS, $20 \mathrm{~g} / \mathrm{L}$ pharmamedia, $\mathrm{pH}$ 7.0), R2YE (103 g/L sucrose, $0.25 \mathrm{~g} / \mathrm{L} \mathrm{K}_{2} \mathrm{SO}_{4}, 10.12 \mathrm{~g} / \mathrm{L} \mathrm{MgCl}_{2} \cdot 6 \mathrm{H}_{2} \mathrm{O}, 10 \mathrm{~g} / \mathrm{L}$ glucose, $0.1 \mathrm{~g} / \mathrm{L}$ Difco Casaminoacids, $0.05 \mathrm{~g} / \mathrm{L} \mathrm{KH}{ }_{2} \mathrm{PO}_{4}, 2.944 \mathrm{~g} / \mathrm{L} \mathrm{CaCl}_{2} \cdot 2 \mathrm{H}_{2} \mathrm{O}, 3 \mathrm{~g} / \mathrm{L}$ L-proline, $5.73 \mathrm{~g} / \mathrm{L}$ TES buffer, $5 \mathrm{~g} / \mathrm{L}$ Difco yeast extract, $5 \mathrm{~mL} 1 \mathrm{~N} \mathrm{NaOH}, 2 \mathrm{~mL}$ trace elements), KM4 (4 g/L glucose, $4 \mathrm{~g} / \mathrm{L}$ yeast extract, $10 \mathrm{~g} / \mathrm{L}$ malt extract, $2 \mathrm{~g} / \mathrm{L} \mathrm{CaCO}_{3}$ ) (12), YEME (3 g/L yeast extract, $3 \mathrm{~g} / \mathrm{L}$ malt extract, $5 \mathrm{~g} / \mathrm{L}$ peptone, $10 \mathrm{~g} / \mathrm{L}$ glucose, $340 \mathrm{~g} / \mathrm{L}$ sucrose) and FR23 (5 g/L glucose, $30 \mathrm{~g} / \mathrm{L}$ soluble starch from potato, $20 \mathrm{~g} / \mathrm{L}$ cottonseed flour, $20 \mathrm{~g} / \mathrm{L}$ cane molasses, $\mathrm{pH} 7$ ) fermentation media. These fermentations were incubated at $28{ }^{\circ} \mathrm{C}, 220 \mathrm{rpm}$ and $70 \%$ humidity for 14 days.

\subsection{Extraction and Detection of Cacaoidin}

Cultures of the recombinant strain S. albus J1074/pCAO, together with the negative control harboring empty pCAP01 vector, were subjected to extraction with acetone 1:1 under continuous shaking at $220 \mathrm{rpm}$ for $2 \mathrm{~h}$. The organic solvent with the secondary metabolites extracted was separated from the biomass via centrifugation, the acetone was removed by a stream of nitrogen overnight and the extracts were resuspended to a final ratio of $20 \% \mathrm{DMSO} /$ water. The resulting microbial extracts were filtered and analyzed using LC-HRESIMS(+)-TOF and MS/MS.

\subsection{Extraction and Detection of Cacaoidin}

Standard solutions of pure cacaoidin at different concentrations were prepared in $20 \%$ DMSO, and a calibration curve was obtained based on the intensity of the LC-MS peak areas (slope $=245491$, Y-intercept $=-6165.4, R^{2}=0.9996$ ). Cacaoidin concentrations from the R2YE culture extracts were determined by interpolating their LC-MS peak areas on the calibration curve $(\mathrm{y}=245941 \mathrm{x}-6165.4)$. 


\section{Conclusions}

Cacaoidin is the first member of the new lanthidin (class V lanthipeptides) RiPP family characterized by the combination of structural features thus far restricted to lanthipeptide and linaridin families and encoded by a new unprecedented RiPP BGC organization that could not be predicted using antiSMASH, BAGEL4 or any other bioinformatic tool. The lack of homology with common lanthionine ring formation or double N-terminal dimethylation enzymes suggests an alternative mechanism of biosynthesis that may be similar to that of the recently described lanthidin lexapeptide. The other unusual structural features of cacaoidin, such as the high number of D-amino acids or the O-glycosylation of tyrosine are supported by the presence in the cao cluster of protein homologues of a $\mathrm{F}_{420} \mathrm{H}_{2}$-dependent oxidoreductase and three glycosyltransferases. The heterologous expression of cacaoidin BGC has demonstrated that the cao cluster contains all the necessary genes to biosynthesize this molecule, and future research is needed to clarify the unassigned functions of the cao genes.

Cacaoidin BGC was only found in the genomes of all publicly available Streptomyces cacaoi subsp. cacaoi strains and not in any other species, suggesting that this cluster may be a species-specific trait. Undoubtedly, cacaoidin BGC has an unprecedented genetic organization, completely different from any other previously described RiPP cluster. Moreover, the detection of similar putative lanthidin homologous clusters opens the door to the study of a new exciting family of RiPPs.

Supplementary Materials: The following are available online at https://www.mdpi.com/article/ 10.3390/antibiotics10040403/s1, Table S1: Primers used for the cloning and checking of the cao biosynthetic gene cluster, Table S2: Closest HHpred homolog for each ORF in cacaoidin BGC, Table S3: BLAST homology between the ORFs in cacaoidin BGC and NCBI found clusters, Figure S1: Alignment the structural amino acidic sequence of cacaoidin with the sequences of other already known lanthipeptides and linaridins, Figure S2: Schematic presentation of the biosynthesis of dTDP-L-rhamnose from D-glucose, Figure S3: Proposed pathway for the $\beta$-L-6-deoxy-gulose sugar biosynthesis for the BLM, TLM and ZMB compounds, Figure S4: Neighbor-joining tree built with Mega X based on nearly complete $16 \mathrm{~S}$ rRNA gene sequences of CA-170360, the 50 closest type strains of the genus Streptomyces and three strains of Streptomyces cacaoi subsp. asoensis.

Author Contributions: F.R.-H., M.S.-H. and O.G. conceived and designed the experiments and wrote the paper. F.R.-H. performed the genetic experiments and bioinformatic analysis. J.M. performed the LC-HRESIMS(+)-TOF and MS/MS experiments. F.J.O.-L. confirmed the structures of the compound and contributed to discussion of the sugar biosynthesis and glycosylation and to writing the paper. All authors have read and agreed to the published version of the manuscript.

Funding: This research was funded by Novo Nordisk Foundation, grant number: NF16OC0021746.

Data Availability Statement: The data presented in this study are openly available in NCBI, GenBank reference number [MT210103]. Publicly available datasets were analyzed in this study. These data can be found in NCBI, GenBank reference numbers [MUBL00000000.1, VSKT00000000.1, JOGD00000000.1, JOHT00000000.1 and BJMM00000000.1].

Acknowledgments: The authors thank Daniel Oves-Costales and Fernando Reyes for helpful advice during the whole process and the Microbiology and Chemistry areas of Fundación MEDINA for the technical support. We thank José Antonio Salas and the University of Oviedo for kindly providing strains Streptomyces albus J1074 and Escherichia coli ET12567/pUB307.

Conflicts of Interest: The authors declare no conflict of interest.

\section{References}

1. Barka, E.A.; Vatsa, P.; Sanchez, L.; Gaveau-Vaillant, N.; Jacquard, C.; Meier-Kolthoff, J.P.; Klenk, H.-P.; Clément, C.; Ouhdouch, Y.; van Wezel, G.P. Taxonomy, Physiology, and Natural Products of Actinobacteria. Microbiol. Mol. Biol. Rev. 2015, 80, 1-43. [CrossRef]

2. Berdy, J. Bioactive microbial metabolites. J. Antibiot. 2005, 58, 1-26. [CrossRef] [PubMed]

3. Genilloud, O. Actinomycetes: Still a source of novel antibiotics. Nat. Prod. Rep. 2017, 34, 1203-1232. [CrossRef] [PubMed]

4. Ventura, M.; Canchaya, C.; Tauch, A.; Chandra, G.; Fitzgerald, G.F.; Chater, K.F.; van Sinderen, D. Genomics of Actinobacteria: Tracing the evolutionary history of an ancient phylum. Microbiol. Mol. Biol. Rev. 2007, 71, 495-548. [CrossRef] [PubMed] 
5. Gomez-Escribano, J.P.; Alt, S.; Bibb, M. Next generation sequencing of actinobacteria for the discovery of novel natural products. Mar. Drugs. 2016, 14, 78. [CrossRef]

6. $\quad$ Blin, K.; Shaw, S.; Steinke, K.; Villebro, R.; Ziemert, N.; Lee, S.Y.; Medema, M.; Weber, T. antiSMASH 5.0: Updates to the secondary metabolite genome mining pipeline. Nucleic Acids Res. 2019, 47, 81-87. [CrossRef]

7. Kautsar, S.A.; Blin, K.; Shaw, S.; Navarro-Muñoz, J.C.; Terlouw, B.R.; van der Hooft, J.J.J.; van Santen, J.A.; Tracanna, V.; Suarez Duran, H.G.; Pascal Andreu, V.; et al. MIBiG 2.0: A repository for biosynthetic gene clusters of known function. Nucleic Acids Res. 2020, 48, 454-458. [CrossRef]

8. van Santen, J.A.; Kautsar, S.A.; Medema, M.H.; Linington, R.G. Microbial natural products databases: Moving forward in the multi-omics era. Nat. Prod. Rep. 2021, 38, 264-278. [CrossRef]

9. Genilloud, O. Mining actinomycetes for novel antibiotics in the omics era: Are we ready to exploit this new paradigm? Antibiotics 2018, 7, 85. [CrossRef]

10. Kloosterman, A.M.; Cimermancic, P.; Elsayed, S.S.; Du, C.; Hadjithomas, M.; Donia, M.S.; Fischbach, M.A.; van Wezel, G.P.; Medema, M.H. Expansion of RiPP biosynthetic space through integration of pan-genomics and machine learning uncovers a novel class of lanthipeptides. PLoS Biol. 2020, 18. [CrossRef] [PubMed]

11. Arnison, P.G.; Bibb, M.J.; Bierbaum, G.; Bowers, A.A.; Bugni, T.S.; Bulaj, G.; Camarero, J.A.; Campopiano, D.J.; Challis, G.L.; Clardy, J.; et al. Ribosomally synthesized and post-translationally modified peptide natural products: Overview and recommendations for a universal nomenclature. Nat. Prod. Rep. 2013, 30, 108-160. [CrossRef] [PubMed]

12. Ortiz-López, F.J.; Carretero-Molina, D.; Sánchez-Hidalgo, M.; Martín, J.; González, I.; Román-Hurtado, F.; de la Cruz, M.; García-Fernández, S.; Reyes, F.; Deisinger, J.P.; et al. Cacaoidin, first member of the new lanthidin RiPP family. Angew. Chem. Int. Ed. 2020, 59, 12654-12658. [CrossRef] [PubMed]

13. Zhang, Q.; Yu, Y.; Velásquez, J.E.; van der Donk, W.A. Evolution of lanthipeptide synthetases. Proc. Natl. Acad. Sci. USA 2012, 109, 18361-18366. [CrossRef] [PubMed]

14. Ma, S.; Zhang, Q. Linaridin natural products. Nat. Prod. Rep. 2020, 37, 1152-1163. [CrossRef]

15. Repka, L.M.; Chekan, J.R.; Nair, S.K.; van der Donk, W.A. Mechanistic understanding of lanthipeptide biosynthetic enzymes. Chem. Rev. 2017, 117, 5457-5520. [CrossRef] [PubMed]

16. Rateb, M.E.; Zhai, Y.; Ehrner, E.; Rath, C.M.; Wang, X.; Tabudravu, J.; Ebel, R.; Bibb, M.; Kyeremeh, K.; Dorrestein, P.C.; et al. Legonaridin, a new member of linaridin RiPP from a Ghanaian Streptomyces isolate. Org. Biomol. Chem. 2015, 13, 9585-9592. [CrossRef]

17. Sit, C.S.; Yoganathan, S.; Vederas, J.C. Biosynthesis of aminovinyl-cysteine-containing peptides and its application in the production of potential drug candidates. Acc. Chem. Res. 2011, 44, 261-268. [CrossRef]

18. Claesen, J.; Bibb, M. Genome mining and genetic analysis of cypemycin biosynthesis reveal an unusual class of posttranslationally modified peptides. Proc. Natl. Acad. Sci. USA 2010, 107, 16297-16302. [CrossRef]

19. Mo, T.; Liu, W.-Q.; Ji, W.; Zhao, J.; Chen, T.; Ding, W.; Yu, S.; Zhang, Q. Biosynthetic insights into linaridin natural products from genome mining and precursor peptide mutagenesis. ACS Chem. Biol. 2017, 12, 1484-1488. [CrossRef]

20. Xu, M.; Cheng, Z.; Bashiri, G.; Wang, J.; Hong, J.; Wang, Y.; Xu, L.; Chen, X.; Huang, S.-X.; Lin, S.; et al. Functional genome mining reveals a novel class $\mathrm{V}$ lanthipeptide containing a D-amino acid introduced by an $\mathrm{F}_{420} \mathrm{H}_{2}$-dependent reductase. Angew. Chem. Int. Ed. 2020, 59, 18029-18035. [CrossRef]

21. Montalbán-López, M.; Scott, T.A.; Ramesh, S.; Rahman, I.R.; van Heel, A.J.; Viel, J.H.; Bandarian, V.; Dittmann, E.; Genilloud, O.; Goto, Y.; et al. New developments in RiPP discovery, enzymology and engineering. Nat. Prod. Rep. 2020, 38, 130-239. [CrossRef]

22. van Heel, A.J.; de Jong, A.; Song, C.; Viel, J.H.; Kok, J.; Kuipers, O.P. BAGEL4: A user-friendly web server to thoroughly mine RiPPs and bacteriocins. Nucleic Acids Res. 2018, 46, 278-281. [CrossRef]

23. Skinnider, M.A.; Merwin, N.J.; Johnston, C.W.; Magarvey, N.A. PRISM 3: Expanded prediction of natural product chemical structures from microbial genomes. Nucleic Acids Res. 2017, 45, 49-54. [CrossRef] [PubMed]

24. Ding, W.; Mo, T.; Mandalapu, D.; Zhang, Q. Substrate specificity of the cypemycin decarboxylase CypD. Synth. Syst. Biotechnol. 2018, 3, 159-162. [CrossRef] [PubMed]

25. Park, Y.; Shin, I.; Rhee, S. Crystal structure of the effector protein HopA1 from Pseudomonas syringae. J. Struct. Biol. 2015, 189, 276-280. [CrossRef]

26. Acedo, J.Z.; Bothwell, I.R.; An, L.; Trouth, A.; Frazier, C.; van der Donk, W.A. O-methyltransferase-mediated incorporation of a $\beta$-amino acid in lanthipeptides. J. Am. Chem. Soc. 2019, 141, 16790-16801. [CrossRef] [PubMed]

27. Huo, L.; Zhao, X.; Acedo, J.Z.; Estrada, P.; Nair, S.K.; van der Donk, W.A. Characterization of a dehydratase and methyltransferase in the biosynthesis of a ribosomally-synthesized and post-translationally modified peptide in Lachnospiraceae. ChemBioChem 2019, 21, 190-199. [CrossRef]

28. Yang, X.; van der Donk, W.A. Post-translational introduction of D-alanine into ribosomally synthesized peptides by the dehydroalanine reductase NpnJ. J. Am. Chem. Soc. 2015, 137, 12426-12429. [CrossRef] [PubMed]

29. Lohans, C.T.; Li, J.L.; Vederas, J.C. Structure and biosynthesis of carnolysin, a homologue of enterococcal cytolysin with D-amino acids. J. Am. Chem. Soc. 2014, 136, 13150-13153. [CrossRef]

30. Huo, L.; van der Donk, W.A. Discovery and characterization of bicereucin, an unusual D-amino acid-containing mixed twocomponent lantibiotic. J. Am. Chem. Soc. 2016, 138, 5254-5257. [CrossRef]

31. Girauld, M.F.; Naismith, J.H. The rhamnose pathway. Curr. Opin. Struct. Biol. 2000, 19, 687-696. [CrossRef] 
32. Galm, U.; Wendt-Pienkowski, E.; Wang, L.; Huang, S.-X.; Unsin, C.; Tao, M.; Coughlin, J.M.; Shen, B. Comparative analysis of the biosynthetic gene clusters and pathways for three structurally related antitumor antibiotics: Bleomycin, tallosomycin and zorbamycin. J. Nat. Prod. 2011, 74, 526-536. [CrossRef]

33. Varki, A.; Cummings, R.D.; Esko, J.D.; Freeze, H.H.; Stanley, P.; Bertozzi, C.R.; Hart, G.W.; Etzler, M.E. Essentials of Glycobiology; Cold Spring Harbor Laboratory Press: New York, NY, USA, 1999.

34. He, H.; Williamson, R.T.; Shen, B.; Graziani, E.I.; Yang, H.Y.; Sakya, S.M.; Petersen, P.J.; Carter, G.T. Mannopeptimycins, novel antibacterial glycopeptides from Streptomyces hygroscopicus, LL-AC98. J. Am. Chem. Soc. 2002, 124, 9729-9736. [CrossRef]

35. Brockhausen, I.; Ho, B.; Liu, B.; Lau, K.; Szarek, W.A.; Wang, L.; Feng, L. Characterization of two $\beta$-1,3-glucosyltransferases from Escherichia coli serotypes O56 and O152. J. Bacteriol. 2008, 190, 4922-4932. [CrossRef] [PubMed]

36. Pradel, E.; Parker, C.T.; Schnaitman, C.A. Structures of the $r f a B, r f a I, r f a J$, and $r f a S$ genes of Escherichia coli K-12 and their roles in assembly of the lipopolysaccharide core. J. Bacteriol. 1992, 174, 4736-4745. [CrossRef] [PubMed]

37. Salcedo, R.G.; Olano, C.; Fernández, R.; Braña, A.F.; Méndez, C.; de la Calle, F.; Salas, J.A. Elucidation of the glycosylation steps during biosynthesis of antitumor macrolides PM100117 and PM100118 and engineering for novel derivatives. Microb. Cell Fact. 2016, 15, 187. [CrossRef] [PubMed]

38. Salem, S.M.; Weidenbach, S.; Rohr, J. Two cooperative glycosyltransferases are responsible for the sugar diversity of saquayamycins isolated from Streptomyces sp. KY 40-1. ACS Chem. Biol. 2017, 12, 2529-2534. [CrossRef]

39. Malmierca, M.G.; Pérez-Victoria, I.; Martín, J.; Reyes, F.; Méndez, C.; Olano, C.; Salas, J.A. Cooperative involvement of glycosyltransferases in the transfer of amino sugars during the biosynthesis of the macrolactam sipanmycin by Streptomyces sp. strain CS149. Appl. Environ. Microbiol. 2018, 84. [CrossRef]

40. Kaunietis, A.; Buivydas, A.; Citavicius, D.J.; Kuipers, O.P. Heterologous biosynthesis and characterization of a glycocin from a thermophilic bacterium. Nat. Commun. 2019, 10, 1115. [CrossRef] [PubMed]

41. Sheng, W.; Xu, B.; Li, Y.; Liu, B.; Wang, H. Substrate tolerance of the biosynthetic enzymes of glycosylated lanthipeptide NAI-112. Org. Biomol. Chem. 2020, 18, 6095-6099. [CrossRef] [PubMed]

42. Plat, A.; Kluskens, L.D.; Kuipers, A.; Rink, R.; Moll, G.N. Requirements of the engineered leader peptide of nisin for inducing modification, export, and cleavage. Appl. Environ. Microbiol. 2011, 77, 604-611. [CrossRef] [PubMed]

43. Knerr, P.J.; van der Donk, W.A. Discovery, biosynthesis, and engineering of lantipeptides. Annu. Rev. Biochem. 2012, 81, 479-505. [CrossRef] [PubMed]

44. Iorio, M.; Sasso, O.; Maffioli, S.I.; Bertorelli, R.; Monciardini, P.; Sosio, M.; Bonezzi, F.; Summa, M.; Brunati, C.; Bordoni, R.; et al. A glycosylated, labionin-containing lanthipeptide with marked antinociceptive activity. ACS Chem. Biol. 2014, 9, 398-404. [CrossRef] [PubMed]

45. Chen, S.; Xu, B.; Chen, E.; Wang, J.; Lu, J.; Donadio, S.; Ge, H.; Wang, H. Zn-dependent bifunctional proteases are responsible for leader peptide processing of class III lanthipeptides. Proc. Natl. Acad. Sci. USA 2019, 116, 2533-2538. [CrossRef] [PubMed]

46. Krawczyk, J.M.; Völler, G.-H.; Krawczyk, B.; Kretz, J.; Brönstrup, M.; Süssmuth, R.D. Heterologous expression and engineering studies of labyrinthopeptins, class III lantibiotics from Actinomadura namibiensis. Chem. Biol. 2013, 20, 111-122. [CrossRef]

47. Geiger, C.; Korn, S.M.; Häsler, M.; Peetz, O.; Martin, J.; Kötter, P.; Morgner, N.; Entian, K.-D. LanI-mediated lantibiotic immunity in Bacillus subtilis: Functional analysis. Appl. Environ. Microbiol. 2019, 85, e00534-19. [CrossRef]

48. Méndez, C.; Salas, J.A. The role of ABC transporters in antibiotics-producing organisms: Drug secretion and resistance mechanisms. Res. Microbiol. 2001, 152, 341-350. [CrossRef]

49. Wood, H.E.; Devine, K.M.; McConnell, D.J. Characterisation of a repressor gene (xre) and a temperature-sensitive allele from the Bacillus subtilis prophage, PBSX. Gene 1990, 96, 83-88. [CrossRef]

50. Cuthbertson, L.; Nodwell, J.R. The TetR family of regulators. Microbiol. Mol. Biol. Rev. 2013, 77, 440-475. [CrossRef] [PubMed]

51. Chen, J.; Xie, J. Role and regulation of bacterial LuxR-like regulators. J. Cell Biochem. 2011, 112, 2694-2702. [CrossRef]

52. Li, Y.; Kong, L.; Shen, J.; Wang, Q.; Liu, Q.; Yang, W.; Deng, Z.; You, D. Characterization of the positive SARP family regulator PieR for improving piericidin A1 production in Streptomyces piomogeues var. Hangzhouwanensis. Synth. Syst. Biotechnol. 2019, 4, 16-24. [CrossRef] [PubMed]

53. Radauer, C.; Lackner, P.; Breiteneder, H. The Bet v1 fold: An ancient, versatile scaffold for binding of large, hydrophobic ligands. BMC Evol. Biol. 2008, 8, 286. [CrossRef] [PubMed]

54. Iyer, L.M.; Koonin, E.V.; Aravind, L. Adaptations of the helix-grip fold for ligand binding and catalysis in the START domain superfamily. Proteins 2001, 43, 134-144. [CrossRef]

55. Ames, B.D.; Korman, T.P.; Zhang, W.; Smith, P.; Vu, T.; Tang, Y.; Tsai, S.-C. Crystal structure and functional analysis of tetracenomycin ARO/CYC: Implications for cyclization specificity of aromatic polyketides. Proc. Natl. Acad. Sci. USA 2008, 105, 5349-5354. [CrossRef] [PubMed]

56. Nan, J.; Brostromer, E.; Liu, X.Y.; Kristensen, O.; Su, X.D. Bioinformatics and structural characterization of a hypothetical protein from Streptococcus mutans: Implication of antibiotic resistance. PLoS ONE 2009, 4, e7245. [CrossRef]

57. Burkhart, B.J.; Hudson, G.A.; Dunbar, K.L.; Mitchell, D.A. A prevalent peptide-binding domain guides ribosomal natural product biosynthesis. Nat. Chem. Biol. 2015, 11, 564-570. [CrossRef]

58. Klinman, J.P.; Bonnot, F. Intrigues and intricacies of the biosynthetic pathways for the enzymatic quinocofactors: PQQ, TTQ, CTQ, TPQ, and LTQ. Chem. Rev. 2014, 114, 4343-4365. [CrossRef] 
59. Latham, J.A.; Iavarone, A.T.; Barr, I.; Juthani, P.V.; Klinman, J.P. PqqD is a novel peptide chaperone that forms a ternary complex with the radical S-adenosylmethionine protein PqqE in the pyrroloquinoline quinone biosynthetic pathway. J. Biol. Chem. 2015, 290, 12908-12918. [CrossRef]

60. Chater, K.F.; Wilde, L.C. Streptomyces albus G mutants defective in the SalGI restriction-modification system. J. Gen. Microbiol. 1980, 116, 323-334. [CrossRef]

61. Jiang, W.; Zhao, X.; Gabrieli, T.; Lou, G.; Ebenstein, Y.; Zhu, T.F. Cas9-assisted targeting of chromosome segments CATCH enables one-step targeted cloning of large gene clusters. Nat. Commun. 2015, 6, 8101. [CrossRef]

62. Yamanaka, K.; Reynolds, K.A.; Kersten, R.D.; Ryan, K.S.; Gonzalez, D.J.; Nizet, V.; Dorrestein, P.C.; Moore, B.S. Direct cloning and refactoring of a silent lipopeptide biosynthetic gene cluster yields the antibiotic taromycin A. Proc. Natl. Acad. Sci. USA 2014, 111, 1957-1962. [CrossRef]

63. Kieser, T.; Bibb, M.J.; Buttner, M.J.; Chater, K.F.; Hopwood, D.A. Practical Streptomyces Genetics; John Innes Foundation: Norwich, UK, 2002; Volume 291.

64. Bilyk, B.; Luzhetskyy, A. Unusual site-specific DNA integration into the highly active pseudo-attB of the Streptomyces albus J1074 genome. Appl. Microbiol. Biotechnol. 2014, 98, 5095-5104. [CrossRef]

65. Tamura, K. Estimation of the number of nucleotide substitutions when there are strong transition-transversion and $\mathrm{G}+\mathrm{C}$-content biases. Mol. Biol. Evol. 1992, 9, 678-687. [PubMed]

66. Saitou, N.; Nei, M. The neighbor-joining method: A new method for reconstructing phylogenetic trees. Mol. Biol. Evol. 1987, 4, 406-425. [CrossRef] [PubMed]

67. Vicente, C.M.; Thibessard, A.; Lorenzi, J.-N.; Benhadj, M.; Hôtel, L.; Gacemi-Kirane, D.; Lespinet, O.; Leblond, P.; Aigle, B. Comparative genomics among closely related Streptomyces strains revealed specialized metabolite biosynthetic gene cluster diversity. Antibiotics 2018, 7, 86. [CrossRef]

68. Seipke, R.F. Strain-level diversity of secondary metabolism in Streptomyces albus. PLoS ONE 2015, 10, e0116457. [CrossRef]

69. Choudoir, M.J.; Pepe-Ranney, C.; Buckley, D.H. Diversification of secondary metabolite biosynthetic gene clusters coincides with lineage divergence in Streptomyces. Antibiotics 2018, 7, 12. [CrossRef] [PubMed]

70. Zhang, J.J.; Yamanaka, K.; Tang, X.; Moore, B.S. Direct cloning and heterologous expression of natural product biosynthetic gene clusters by transformation-associated recombination. Methods Enzymol. 2019, 621, 87-110. [CrossRef] [PubMed]

71. Koren, S.; Walenz, B.P.; Berlin, K.; Miller, J.R.; Bergman, N.H.; Phillippy, A.M. Canu: Scalable and accurate long-read assembly via adaptive k-mer weighting and repeat separation. Genome Res. 2017, 27, 722-736. [CrossRef] [PubMed]

72. Walker, B.J.; Abeel, T.; Shea, T.; Priest, M.; Abouelliel, A.; Sakthikumar, S.; Cuomo, C.A.; Zeng, Q.; Wortman, J.; Young, S.K.; et al. Pilon: An Integrated Tool for Comprehensive Microbial Variant Detection and Genome Assembly Improvement. PLoS ONE 2014, 9, e112963. [CrossRef]

73. Agrawal, P.; Khater, S.; Gupta, M.; Sain, N.; Mohanty, D. RiPPMiner: A bioinformatics resource for deciphering chemical structures of RiPPs based on prediction of cleavage and cross-links. Nucleic Acids Res. 2017, 45, W80-W88. [CrossRef]

74. Johnson, M.; Zaretskaya, I.; Raytselis, Y.; Merezhuk, Y.; McGinnis, S.; Madden, T.L. NCBI BLAST: A better web interface. Nucleic Acids Res. 2008, 36, W5-W9. [CrossRef] [PubMed]

75. Zimmermann, L.; Stephens, A.; Nam, S.-Z.; Rau, D.; Kübler, J.; Lozajic, M.; Gabler, F.; Söding, J.; Lupas, A.N.; Alva, V. A Completely Reimplemented MPI Bioinformatics Toolkit with a New HHpred Server at its Core. J. Mol. Biol. 2018, 2836, 30587-30589. [CrossRef]

76. Jian, W.; Zhu, T.F. Targeted isolation and cloning of 100-kb microbial genomic sequences by Cas9-assisted targeting of chromosome segments. Nat. Protoc. 2016, 11, 960-975. [CrossRef] [PubMed]

77. Tong, Y.; Robertsen, H.L.; Blin, K.; Weber, T.; Lee, S.Y. CRISPR-Cas9 Toolkit for Actinomycete Genome Editing. Methods Mol. Biol. 2018, 1671, 163-184. [CrossRef] [PubMed] 